\title{
Scaling Behavior of the First Arrival Time of a Random-Walking Magnetic Domain
}

\author{
M.-Y. Im, ${ }^{1,2}$ S.-H. Lee, ${ }^{2}$ D.-H. Kim, ${ }^{3}$ P. Fischer, ${ }^{1}$ and S.-C. Shin $^{2 *}$ \\ ${ }^{1}$ Center for X-ray Optics, Lawrence Berkeley National Laboratory, Berkeley CA94720, \\ USA; \\ ${ }^{2}$ Department of Physics and Center for Nanospinics of Spintronic Materials, Korea \\ Advanced Institute of Science and Technology, Daejeon 305-701, Korea; \\ ${ }^{3}$ Department of Physics, Chungbuk National University, Chungbuk, Korea
}

\begin{abstract}
We report a universal scaling behavior of the first arrival time of a traveling magnetic domain wall into a finite space-time observation window of a magneto-optical microscope enabling direct visualization of a Barkhausen avalanche in real time. The first arrival time of the traveling magnetic domain wall exhibits a nontrivial fluctuation and its statistical distribution is described by universal power-law scaling with scaling exponents of $1.34 \pm 0.07$ for $\mathrm{CoCr}$ and $\mathrm{CoCrPt}$ films, despite their quite different domain evolution patterns. Numerical simulation of the first arrival time with an assumption that the magnetic domain wall traveled as a random walker well matches our experimentally observed scaling behavior, providing an experimental support for the random-walking model of traveling magnetic domain walls.
\end{abstract}


The statistical distribution of the arrival time into finite area in seemingly random dynamical systems has been extensively investigated in a wide variety of physical systems, such as queuing of server jobs, earthquakes, epidemic, ocean waves, and heavy vehicle traffic [1-7]. Magnetization reversal under an external magnetic field has been considered to be a very good example of a system with complex dynamics, exhibiting non-trivial fluctuations leading to scaling behavior, ever since the almost century-old discovery of the Barkhausen avalanche [8-10]. Most statistical studies on magnetic reversal phenomena have been concentrated to examine fluctuating quantities such as the size of a Barkhausen jump, which exhibits scaling behavior described by a powerlaw distribution of the fluctuating quantity [8-14]. However, a long-standing unsolved issue is the statistical behavior of the first arrival time of a traveling magnetic domain wall, which presumably undergoes a chain of avalanche events until it finally enters the finite space-time observation window. But, there has been no experimental study devoted to the statistical behavior of first arrival times of a traveling magnetic domain wall during a Barkhausen avalanche in ferromagnetic films.

Traveling domain wall motion during a Barkhausen avalanche has long been predicted to have a random-walking behavior [15-18]. It is believed that pinning sites with a Gaussian spatial distribution provide a playground for the random-walking magnetic domain wall. Much effort has been devoted to understanding the Barkhausen avalanche, for example based on the Langevin equation [19] with a Brownian-motionlike randomly fluctuating force. Recently, great attention has been paid to the scaling behavior of random-walking statistics [20-23], with various scaling exponents theoretically predicted depending on the details of the random-walking method. So far, very little connection has been made between the theoretically predicted random walk behavior of a Barkhausen avalanche and experimentally observed fluctuating magnetic domain walls. Thus, providing clear experimental evidence for the random-walking magnetic domain wall model and for the scaling behavior of magnetic domain dynamics remains a scientific challenge to date.

In this Letter we have investigated the statistical distribution of the first arrival time 
of a traveling magnetic domain wall into an observation area during a Barkhausen avalanche. The samples used in our study were nanogranular $\mathrm{CoCr}$ and $\mathrm{CoCrPt}$ alloy films, which show quite different domain evolution patterns even for small additions of Pt $[24,25]$.

The magnetic domain walls were triggered by applying an external magnetic field near the coercive field strength, to $1 \mathrm{~cm} \times 1 \mathrm{~cm} \mathrm{CoCr}$ and $\mathrm{CoCrPt}$ alloy films. The distribution of the first arrival times of the domain walls into the $40 \mu \mathrm{m} \times 32 \mu \mathrm{m}$ observation area was measured using thousands of time-resolved domain evolution patterns with observation time windows of 1, 30, and 60 seconds. Direct observation of time-resolved magnetic domain patterns was carried out using a magneto-optical microscope magnetometer (MOMM)[12]. The MOMM system basically consists of a polarizing optical microscope designed to observe in-plane magnetic domains via the longitudinal magnetic-optical Kerr effect. The spatial resolution was $400 \mathrm{~nm}$ and the Kerr angle resolution was $0.1^{\circ}$. The temporal resolution was about $30 \mathrm{~ms}$ and a series of 128 domain evolution patterns was captured in real time. The time-resolved domain images were observed after applying a reversing magnetic field to an initially saturated sample. To avoid being affected by the difference in the field-sweeping rates, the strength of the applied magnetic field was kept constant near the coercive field strength $\left(\mathrm{H}_{\mathrm{c}}\right) \cdot \mathrm{Co}_{82} \mathrm{Cr}_{18}$ and $\left(\mathrm{Co}_{82} \mathrm{Cr}_{18}\right)_{97} \mathrm{Pt}_{3}$ alloy films were prepared on glass substrates at an ambient temperature using dc magnetron co-sputtering of a $\mathrm{CoCr}$ alloy target and a $\mathrm{Pt}$ target at a base pressure of $8 \times 10^{-7}$ Torr and an Ar sputtering pressure of 3 mTorr. The thickness of all samples was controlled to be $10 \mathrm{~nm}$ in order to obtain two-dimensional magnetic domain dynamics. The composition of the $\mathrm{CoCr}$ was chosen to be $82: 18$ since the segregation of $\mathrm{Cr}$ into the grain boundaries is known to be maximal at this composition, providing a sufficient number of pinning sites for the traveling magnetic domain wall [26]. The compositions of the alloy samples were confirmed by energy dispersive $\mathrm{x}$-ray spectroscopy. The magnetic easy axis determined using a torque magnetometer was found to be in the plane for all samples.

Fig. 1 (a) shows the time-resolved domain evolution patterns of the $\mathrm{CoCr}$ alloy film 
obtained from five consecutive experiments using the MOMM system, together with time-dependent magnetization reversal curves corresponding to the domain evolution patterns. The color coding from red to blue indicates an elapsed time between 0 to 1 second. The discrete and random Barkhausen avalanches are clearly visible as shown in each row in Fig. 1(a). We specify the time when the Barkhausen jump was observed in the upper left corner of each picture. The images were obtained successively from the same $40 \mu \mathrm{m} \times 32 \mu \mathrm{m}$ area of film under a constant reversing magnetic field with strength about $99 \%$ of the coercivity $\mathrm{H}_{\mathrm{c}}(\sim 85 \mathrm{Oe})$. Thus, the range of the observation window in Fig. 1(a) was $40 \mu \mathrm{m} \times 32 \mu \mathrm{m}$ spatially and 1 second temporally. Considering a total sample area of $1 \mathrm{~cm} \times 1 \mathrm{~cm}$, the area of the observation window was smaller than the total area of the system by the order of $10^{5}$. Interestingly, one clearly sees in Fig. 1(a) that the first arrival time of the magnetic domain wall into the finite observation window is quite different for successive measurements although the observations were carried out under identical experimental conditions.

The difference in the first arrival time of the magnetic domain wall can also be clearly seen in the time-dependent magnetization reversal curves. The magnetization curves were determined by analyzing the time-resolved domain images. The magnetization of each magnetic domain was either parallel or antiparallel to the applied magnetic field direction, which could be seen by two clear levels of magnetic contrast for all the samples. The magnetization curve was thus determined by quantitatively analyzing the magnetic domain area of each image, neglecting magnetization variation perpendicular to the plane of the film. The magnetization curve with sudden changes of magnetization is typical of the nature of a Barkhausen avalanche. The first arrival time of the magnetic domain wall into the observation area of the MOMM system is denoted as $\mathrm{T}_{0}$ and the measured value of $\mathrm{T}_{0}$ for each experiment is indicated in the second column of Fig. 1(a). It shows that $\mathrm{T}_{0}$ fluctuates for each successive measurement even under the same experimental conditions. Considering the fact that the $\mathrm{CoCr}$ alloy film provides numerous pinning sites and segregated grains with the average size around 20 $\mathrm{nm}$ due to the higher $\mathrm{Cr}$ composition at grain boundaries [24-28], one can hypothesize 
that the magnetic domain walls jump around among these pinning sites, generating the Barkhausen avalanches, and finally arrive into the experimental observation area with a different first arrival time for each observation. To verify this hypothesis, the images of the first-arriving magnetic domain walls are compared. In Fig. 1(b), several images of the first arriving domain walls are superimposed in one picture. Although the overall domain shapes are always spike-like, there are only a few common positions where the first arriving domain walls are repeatedly found. These common positions are probably due to the existence of strong local pinning sites as indicated by the arrows.

To systematically investigate the statistical nature of $T_{0}$, the experiments have been repeated more than 1000 times at each observation area and on various areas of the same $\mathrm{Co}_{82} \mathrm{Cr}_{18}$ film. Three different time windows of 1, 30, and 60 seconds have been used and the distribution of $\mathrm{T}_{0}$ is plotted on a log-log scale in Fig. 2(a). The insets of the figure are typical examples of time-resolved domain evolution pattern of the $\mathrm{CoCr}$ film. The first arrival time $T_{0}$ of the traveling magnetic domain wall in a Barkhausen avalanche shows a non-trivial fluctuation and distributions of $\mathrm{T}_{0}$ taken at observation time windows of 1,30 , and 60 seconds can be described by a power-law scaling distribution $\mathrm{P}\left(\mathrm{T}_{0}\right) \sim\left(\mathrm{T}_{0}\right)^{-\tau}$ with $\tau=1.32 \pm 0.05, \tau=1.34 \pm 0.06$, and $\tau=1.33 \pm 0.07$, respectively. The scaling exponent is found to be the same with a value about 1.33 within the measurement error independent of the length of the observation time window. We have confirmed that the value of the scaling exponents of about 1.33 were nearly same in distributions taken at the applied magnetic field of $0.97 \mathrm{H}_{\mathrm{c}}, 0.98 \mathrm{H}_{\mathrm{c}}$ and 0.997 $\mathrm{H}_{\mathrm{c}}$. Therefore, the first arrival time of a magnetic domain wall is believed to be a universal behavior in different field strength.

We also investigated the statistical distribution of $\mathrm{T}_{0}$ for $\left(\mathrm{Co}_{82} \mathrm{Cr}_{18}\right)_{97} \mathrm{Pt}_{3}$ alloy film. Typical examples of the time-resolved domain evolution pattern of $\left(\mathrm{Co}_{82} \mathrm{Cr}_{18}\right)_{97} \mathrm{Pt}_{3}$ are shown in the inset of Fig. 2(b). The $\left(\mathrm{Co}_{82} \mathrm{Cr}_{18}\right)_{97} \mathrm{Pt}_{3}$ film has a much simpler stripe-like domain wall evolution pattern, compared to the spike-like one observed in the $\mathrm{Co}_{82} \mathrm{Cr}_{18}$ film. It has been predicted that only $3 \%$ addition of Pt in atomic composition could significantly reduce the $\mathrm{Cr}$ segregation at grain boundaries, possibly reducing the 
number of defects and hence simplifying the domain shapes [24,27]. The distribution of $\mathrm{T}_{0}$ for the $\left(\mathrm{Co}_{82} \mathrm{Cr}_{18}\right)_{97} \mathrm{Pt}_{3}$ film was determined in the same way as for the $\left(\mathrm{Co}_{82} \mathrm{Cr}_{18}\right)$ film under the field strength about $99 \%$ of coercivity ( $~ 92$ Oe) for CoCrPt alloy film. In Fig. 2(b), the distribution of $\mathrm{T}_{0}$ for the $\left(\mathrm{Co}_{82} \mathrm{Cr}_{18}\right)_{97} \mathrm{Pt}_{3}$ alloy film is plotted on a log-log scale putting together the distributions with time windows of 1, 30, and 60 seconds. We again observe power-law distributions, with scaling exponents of $1.36 \pm 0.04,1.35 \pm$ 0.05 , and $1.34 \pm 0.03$ for each time window, as shown in the figure. Note that the experimentally determined values of the scaling exponents for the $\mathrm{CoCr}$ and $\mathrm{CoCrPt}$ alloy films are nearly same within the experimental error.

To theoretically investigate the scaling behavior of the first arrival time of a magnetic domain wall, we consider a random walk as an analogy to the moving domain wall. It has been conjectured in numerous theoretical studies over the past decades that a domain wall behaves like a random-walking single particle [16, 17, 19]. Simulations have been carried out to determine the first arrival time of an unbiased random-walker in two dimensions with a Gaussian distributed step length with mean values $\mu=1,2,4$ and standard deviations $\sigma=1,2,3$. The step length of the random-walker can be considered as the distance between two successive pinning sites. In our simulation, we have applied the simple model of an unbiased 2D random-walker to CoCrPt alloy film with a simpler domain wall pattern of stripe-like compared to one of $\mathrm{CoCr}$ alloy film, because domain walls of $\mathrm{CoCrPt}$ alloy film has been observed to be propagated with $2 \mathrm{D}$ degree of freedom of motion in large numbers of domain evolution patterns directly observed by MOMM, as shown in inserted images of Fig. 2 (b), where one clearly sees that domain walls with various angles and shapes are evolved in all direction on the sample. We found that our random walk simulation code produces a scaling behavior of $\left\langle\mathrm{R}_{\mathrm{N}}^{2}\right\rangle \sim \mathrm{N}^{2 \mathrm{v}}$ with a typical scaling exponent $\mathrm{v}=0.5$, where $\mathrm{R}_{\mathrm{N}}$ is the displacement of the random-walker from the starting point after $\mathrm{N}$ steps. The value of the scaling exponent $v=0.5$ has been reported in numerous studies $[29,30]$. In this simulation, 10 random walkers were generated in the area of $2000 \times 2000$ cells. The observation area of $20 \times 20$ cells was set with consideration of the ratio between the sample size $(1 \mathrm{~cm} \times$ 
$1 \mathrm{~cm})$ of the $\mathrm{CoCr}$ and $\mathrm{CoCrPt}$ films and the observation window size $(40 \mu \mathrm{m} \times 32 \mu \mathrm{m})$ of the MOMM. The initial position of each random-walker was randomly chosen but constrained to be outside the observation area. The direction of the random walk step in two dimensions was random without any preferred direction. The random-walking process was iterated until one of the 10 random-walkers entered the observation area. Thus, the number of steps during this process corresponds to the first arrival time of the magnetic domain wall.

The statistical distribution of the first arrival time was determined by running more than 50,000 simulations for each choice of $\mu$ and $\sigma$, as shown in Fig. 3. The Gaussian distribution functions used in our simulations are inserted in the figures. One clearly sees that the first arrival time of the random-walker in all cases displays a power-law scaling distribution, in agreement with the experimental results for the first arrival time of a traveling magnetic domain wall. Furthermore, the scaling exponent determined from our simulation, considered to be the most important parameter in the description of this non-trivial fluctuation phenomenon, is found to be the same with a value of $1.35 \pm$ 0.06 for all configurations irrespective of the values of $\mu$ and $\sigma$. Our simulation results seem to be very well matched to the experimental ones within the errors, which answers the historical question of whether a traveling magnetic domain wall can be described by a random walk.

This results report for the first time the direct experimental observation of the scaling behavior of the first arrival time of a magnetic domain wall into a finite window of space and time in $\mathrm{CoCr}$ and $\mathrm{CoCrPt}$ alloy films. The travelling magnetic domain wall during a Barkhausen avalanche appears to exhibit a scaling statistical distribution with the same scaling exponent of about 1.34 in both systems, despite their different domain evolution patterns. Very interestingly, the experimental value of the scaling exponent coincides with the theoretical one determined from a random-walking model of a travelling magnetic domain wall. Therefore, the present study supports a long-standing, but until now experimentally unproven, conjecture that a moving domain wall can be considered as a random-walker. 


\section{Acknowledgements}

This work was supported by MOST through the National Research Lab. Project and the Cavendish-KAIST Research Cooperation Project, and KAIST through the Leading Basic S\&T Research Project. This work was also supported by the research grant of the Chungbuk National University in 2007 and by the Korea Research Foundation Grant funded by the Korean Government (MOEHRD, Basic Research Promotion Fund) (KRF-2007-331-C0097). M-Y Im and P. Fischer acknowledge support by the Director,

Office of Science, Office of Basic Energy Sciences, Materials Sciences and Engineering Division, of the U.S. Department of Energy under Contract No. DE-AC02-05CH11231. 


\section{References}

[1]. P. A. Houle and J. P. Sethna, Phys. Rev. E 54, 278 (1996).

[2]. P. Miltenberger, D. Sornette, and C. Vanette, Phys. Rev. Lett. 71, 3604 (1993).

[3]. D. P. Smethurst and H. C. Williams, Nature 410, 652 (2001).

[4]. C. J. Rhodes and R. M. Anderson, Nature 381, 600 (1996).

[5]. D. Brockmann, L. Hufnagel, and T. Geisel, Nature 439, 462 (2006).

[6]. L. S. Liebovitch and I. B. Schwartz1, Phys. Rev. E. 68, 017101-1 (2003).

[7]. R. P. Cowburn, Science 311,183 (2006).

[8]. H. Barkhausen, Z. Phys. 20, 401 (1919).

[9]. J. Sethna, K. A. Dahmen, and C. R. Myers, Nature 410, 242 (2001).

[10]. G. Durin and S. Zapperi, Phys. Rev. Lett. 84, 4075 (2000).

[11]. S. Zapperi, P. Cizeau, G. Durin, and H. E. Stanley, Phys. Rev. B 58, 6353 (1998).

[12]. D.-H. Kim, S.-B. Choe, and S.-C. Shin, Phys. Rev. Lett. 90, 87203-1 (2003).

[13]. A. Schwarz, M. Liebmann, U. Kaiser, and R. Wiesendanger, Phys. Rev. Lett. 92, 077206-1 (2004).

[14]. M. R. Freeman and B. C. Choi, Science 294, 1484 (2001).

[15]. S. Takesue, T. Mitsudo, and H. Hayakawa, Phys. Rev. E 68, 015103-1 (2003).

[16]. Y. Wada and J. R. Schrieffer, Phys. Rev. B 18, 3897 (1978).

[17]. H. Scher and M. Lax, Phys. Rev. B 7, 4491 (1973).

[18]. A. Baldassarri, F. Dalton, A. Petri, S. Zapperi, G. Pontuale, and L. Pietronero, Phys. Rev. Lett. 96, 118002-1 (2006).

[19]. B. Alessandro, C. Beatrice, G. Bertotti, and A. Montorsi, J. Appl. Phys. 68, 2901 (1990).

[20]. M. F. Shlesinger, J. Klafter, and Y. M. Wong, J. Stat. Phys. 27, 499 (1982).

[21]. J. D. Noh and H. Rieger, Phys. Rev. Lett. 92, 118701-1 (2004).

[22]. M. Ding and W. Yang, Phys. Rev. E 52, 207 (1995).

[23]. A. Dobay, J. Dubochet, K. Millett, P. E. Sottas, and A. Stasiak, PNAS 100 (10), $5611(2003)$.

[24]. M.-Y. Im, D.-H. Kim, and S.-C. Shin, Phys. Rev. B, in preparation.

[25]. J. W. Smits, S. B. Luitjens, and F. J. A. den Broeder, J. Appl. Phys. 55(6), 2260 (1984).

[26]. K. Kimoto, Y. Yahisa, T. Hirano, K. Usami, and S. Narishige, Jpn. J. Appl. Phys. 34, 352 (1995).

[27]. N. Inaba and M. Futamoto, J. Appl. Phys. 87, 6863 (2000). 
[28]. M. - Y. Im, D. -H. Kim, and S. -C. Shin, Phys. Rev. B 72, 132416 (2005).

[29]. J. W. Halley, H. Nakanishi, and R. Sundararajan, Phys. Rev. B 31, 293 (1985).

[30]. E. P. Raposo, S. M. de Oliveira, A. M. Nemorovsky, and M. D. Coutinho-Filho, J. Phys. 59, 633-645 (1991). 


\section{Figure Captions}

FIG. 1. (a) Time-resolved domain evolution patterns and corresponding time-dependent magnetization reversal curves taken from five consecutive experiments at the same sample area $(40 \mu \mathrm{m} \times 32 \mu \mathrm{m})$ of $\mathrm{Co}_{82} \mathrm{Cr}_{18}$ alloy film. The color code represents the elapsed time from 0 to $1 \mathrm{sec}$. The sample was initially saturated and then a reversing field of $99 \%$ of $\mathrm{H}_{\mathrm{c}}$ was applied to trigger a magnetic domain wall. (b) Superimposed image of first arriving magnetic domain walls, where the colors of domain walls correspond to the elapsed time represented by the color palette in the figure.

FIG. 2. Distributions of the first arrival time of a magnetic domain wall in (a) $\mathrm{Co}_{82} \mathrm{Cr}_{18}$ and (b) $\left(\mathrm{Co}_{82} \mathrm{Cr}_{18}\right)_{97} \mathrm{Pt}_{3}$ alloy films determined from the statistical analysis of the fluctuating first arrival time. Best fitting curves determined by the least mean square method are shown in the figures. The inserted images are typical time-resolved domain evolution patterns of $\mathrm{Co}_{82} \mathrm{Cr}_{18}$ and $\left(\mathrm{Co}_{82} \mathrm{Cr}_{18}\right)_{97} \mathrm{Pt}_{3}$ alloy films.

FIG. 3. Distributions of the first arrival time of a random walker obtained from numerical simulations with varying (a) mean values $(\mu=1,2,4)$ and (b) standard deviations $(\sigma=1,2,3)$ of the Gaussian distributed random-walk step size. Best fitting curves are displayed in the figures. 


\section{$\mathbf{a}$}

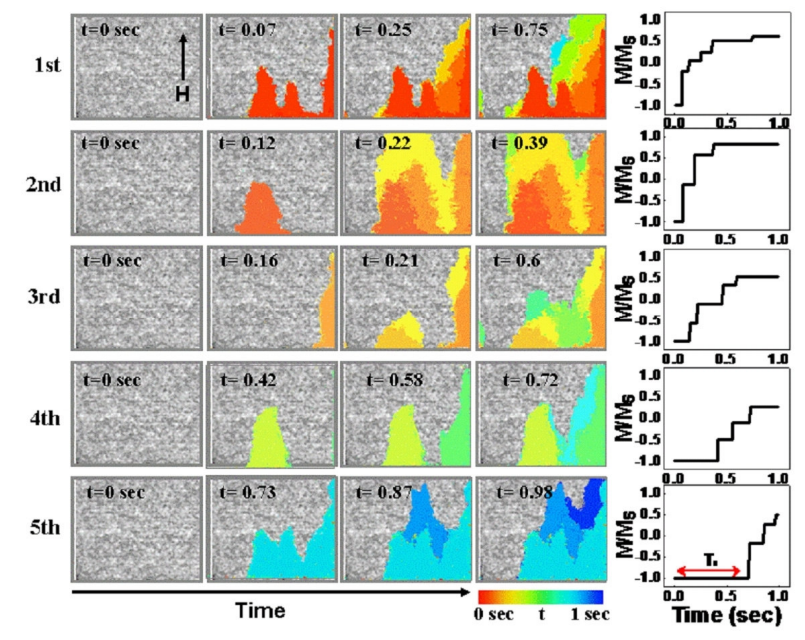

b

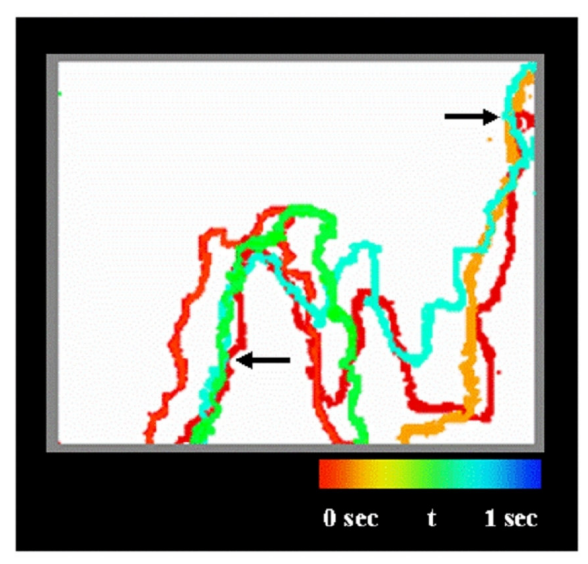

FIG. 1 
$\mathbf{a}$

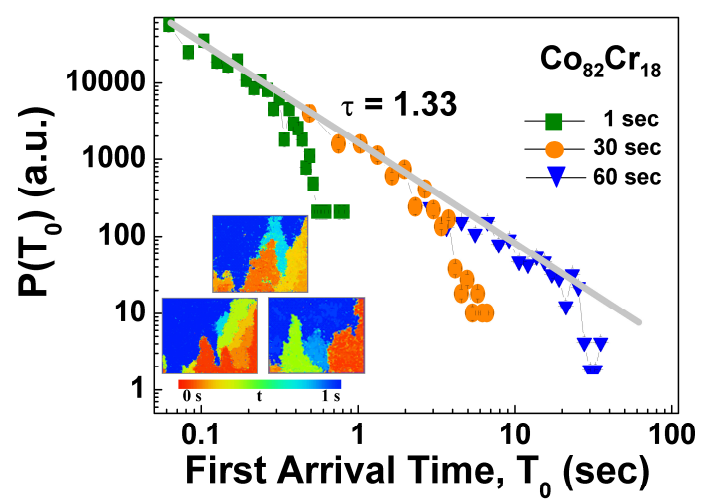

b

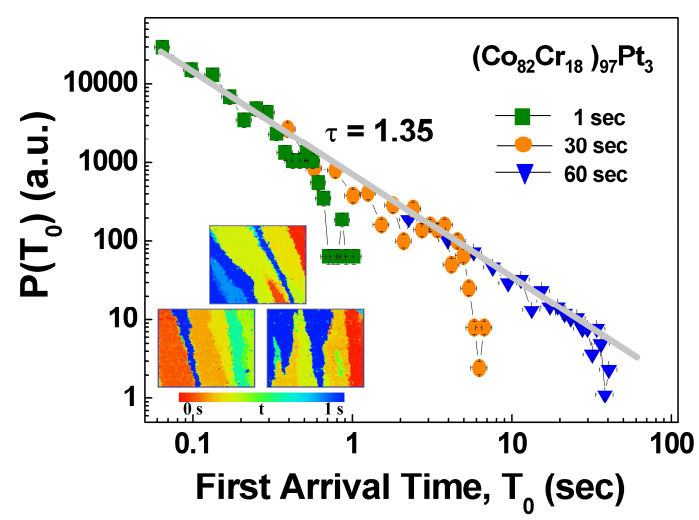

FIG. 2 
a

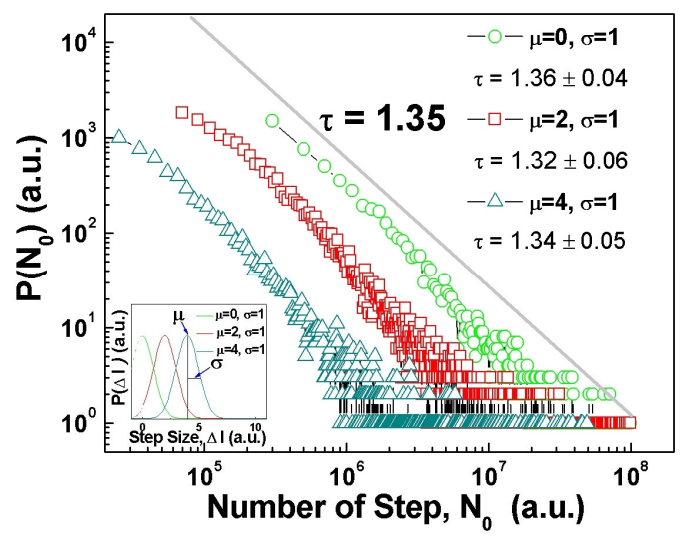

b

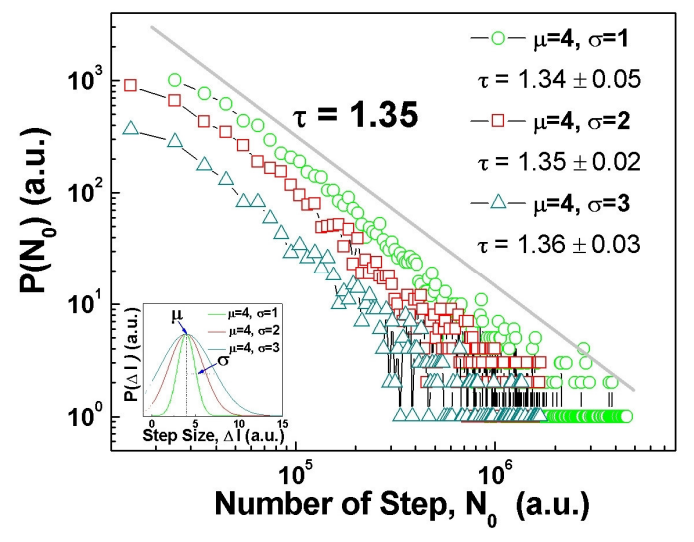

FIG. 3 